\title{
Association between dietary fat intake and insulin resistance in Chinese child twins
}

\author{
Tao Huang ${ }^{1,2 *}$, Terri Beaty ${ }^{3}, \mathrm{Ji} \mathrm{Li}^{2}$, Huijuan Liu ${ }^{4}$, Wei Zhao ${ }^{4}$ and Youfa Wang ${ }^{2,3,5 *}$ \\ ${ }^{1}$ Epidemiology Domain, Saw Swee Hock School of Public Health and Department of Medicine, Yong Loo Lin School of \\ Medicine, National University of Singapore, Singapore, 021800 \\ ${ }^{2}$ Department of International Health, Johns Hopkins Bloomberg School of Public Health, Baltimore, MD 21205, USA \\ ${ }^{3}$ Department of Epidemiology, Johns Hopkins Bloomberg School of Public Health, Baltimore, MD 21205, USA \\ ${ }^{4}$ Jiaxing Maternal and Child Health Hospital, Jiaxing, Zhejiang 314051, People's Republic of China \\ ${ }^{5}$ Department of Epidemiology and Environmental Health, School of Public Health and Health Professions, University at \\ Buffalo, State University of New York, Buffalo, NY 14228, USA
}

(Submitted 29 April 2016 - Final revision received 26 November 2016 - Accepted 6 December 2016 - First published online 25 January 2017)

\section{Abstract}

Dietary fat intake is correlated with increased insulin resistance (IR). However, it is unknown whether gene-diet interaction modulates the association. This study estimated heritability of IR measures and the related genetic correlations with fat intake, and tested whether dietary fat intake modifies the genetic influence on type 2 diabetes (T2D)-related traits in Chinese child twins. We included 622 twins aged 7-15 years (n 311 pairs, 162 monozygotic (MZ), 149 dizygotic (DZ)) from south-eastern China. Dietary factors were measured using FFQ. Structural equation models were fit using Mx statistical package. The intra-class correlation coefficients for all traits related to T2D were higher for MZ twins than for DZ twins. Dietary fat and fasting serum insulin (additive genetic correlation $\left(r_{\mathrm{A}}\right)$ 0.20; $95 \%$ CI 0.08, 0.43), glucose $\left(r_{\mathrm{A}} 0 \cdot 12 ; 95 \%\right.$ CI $0.01,0 \cdot 40)$, homoeostasis model of assessment-insulin resistance (Homa-IR) $\left(r_{\mathrm{A}} 0 \cdot 22 ; 95 \%\right.$ CI $\left.0 \cdot 10,0 \cdot 50\right)$ and the quantitative insulin sensitivity check index (Quicki) $\left(r_{\mathrm{A}}-0 \cdot 22 ; 95 \% \mathrm{CI}-0 \cdot 40,0 \cdot 04\right)$ showed strong genetic correlations. Heritabilities of dietary fat intake, fasting glucose and insulin were estimated to be 52,70 and $70 \%$, respectively. More than $70 \%$ of the phenotypic correlations between dietary fat and insulin, glucose, Homa-IR and the Quicki index appeared to be mediated by shared genetic influence. Dietary fat significantly modified additive genetic effects on these quantitative traits associated with T2D. Analysis of Chinese twins yielded high estimates of heritability of dietary fat intake and IR. Genetic factors appear to contribute to a high proportion of the variance for both insulin sensitivity and IR. Dietary fat intake modifies the genetic influence on blood levels of insulin and glucose, Homa-IR and the Quicki index.

Key words: Insulin sensitivity: Insulin resistance: Diabetes: Gene-diet interactions: Diet: Fat intake: Children

Type 2 diabetes (T2D) is characterised by the loss of $\beta$ cell function and insulin sensitivity (IS), and is a multifactorial abnormality with a strong genetic component to its aetiology; however, there are several recognised environmental influences as well ${ }^{(1)}$. Epidemiological studies and clinical trials have examined the roles of lifestyle and dietary factors (e.g. fat intake) in diabetes prevention ${ }^{(2,3)}$. Total fat and SFA intakes were positively associated with T2D risk $^{(4)}$. It has been suggested that insulin resistance (IR) is a postprandial phenomenon linked to acute dietary fat metabolism ${ }^{(5)}$. Animal studies have demonstrated that the type of fat in the diet affects IS by changing the fatty acid composition of membrane lipids ${ }^{(6)}$. Such phenomena support findings of studies linking the nature of dietary fats to dysfunctions in insulin secretion and increased risk of $\mathrm{T} 2 \mathrm{D}^{(4,7-9)}$.
Recent genome-wide association studies have identified several genes associated with T2D and glucose and/or insulin levels ${ }^{(10,11)}$. These individual variants confer relatively small increments in risk, however, and when combined explain only a small proportion of familial clustering, leading many to question how the remaining 'missing' heritability can be explained $^{(12)}$. It is proposed that gene-environment interaction may explain the missing heritability of $\mathrm{T} 2 \mathrm{D}^{(12)}$. In the past decade, gene-gene and gene-environment interactions have documented to play critical roles in the aetiology of diabetes ${ }^{(1)}$. A growing body of research indicates that dietary fat may modify the genetic association with T2D-related traits ${ }^{(13-17)}$.

However, it remains unclear to what extent dietary fat intake could influence insulin secretion and resistance, and much less

Abbreviations: DZ, dizygotic; Homa-IR, homoeostasis model of assessment-insulin resistance; IR, insulin resistance; IS, insulin sensitivity; MZ, monozygotic; Quicki, quantitative insulin sensitivity check index; $r_{\mathrm{A}}$, additive genetic correlation; T2D, type 2 diabetes.

* Corresponding authors: T. Huang, email huang_tao@nuhs.edu.sg; Y. Wang, email youfawang@gmail.com 
is known about the relationship between dietary fat and diabetes in children, compared with adults, especially during puberty and in developing countries. This study aimed to estimate the genetic and environmental influence on dietary fat intake, IS and IR as well as to determine the correlations among these phenotypes using data collected from Chinese child twins.

\section{Methods \\ Study sample and data collection}

We used the baseline data collected from 622 twins aged 7-15 years ( $n 311$ pairs, monozygotic (MZ):dizygotic $(\mathrm{DZ}) \approx 1: 1$, male:female $\approx 1: 1$ ) at Jiaxing, Zhejiang Province, south-eastern China during 2009. The study protocol was reviewed and approved by the Institutional Review Boards of The Johns Hopkins University Bloomberg School of Public Health and Jiaxing Maternity and Child Health Care Hospital in Jiaxing, China.

All twins and their mothers visited one of three clinics closest to their home on a designated weekend morning for data collection. Physical and medical examinations, including collection of fasting blood samples, were performed by research physicians and nurses with specific training for this study. Instruments were calibrated before use at each session. Mothers completed a questionnaire about their family background and about their children.

\section{Anthropometric measures}

Subjects were measured by trained research staff following standard protocols. Barefoot height (in $\mathrm{cm}$ to nearest $0 \cdot 1$ ) was measured using a wall-mounted stadiometer (Yiwu Fengkuang); two readings were recorded and averaged for analysis. Participants were weighed without shoes and with light clothes to the nearest $0 \cdot 1 \mathrm{~kg}$ using a digital scale integrated into a bioelectric impedance analyzer system (Yiwu Fengkuang). BMI was calculated as weight (kg)/height $(\mathrm{m})^{2}$.

\section{Dietary intake assessment}

Food consumption was assessed using a 145-item, selfadministered FFQ, which measured children's food intakes during the previous 12 months. This FFQ was initially designed for children in Beijing and was validated against four 24-h recalls; the FFQ showed good reliability and moderate validity ${ }^{(18)}$. Subjects provided information on the type and amount of food consumed. Children were also provided with two-dimensional colourful pictures of measuring plates and bowls to facilitate estimation of portion size. Standardised interviews were conducted when necessary to help children better understand the questionnaire. All interviewers were required to follow a standardised protocol for data collection. Energy and nutrient intakes were calculated using the Nutrition Data Systems established by the China Centers for Disease Control and Prevention ${ }^{(19)}$.

\section{Assessment of fasting serum glucose, insulin and basal insulin resistance and sensitivity measurements}

Fasting serum glucose was measured by the glucose oxidase method. Insulin was examined by electrochemiluminescence immunoassay. Homoeostasis model of assessment-insulin resistance (Homa-IR) and the quantitative insulin sensitivity check index (Quicki) were calculated on the basis of the following formulae: HOMA-IR $=$ fasting insulin $(\mu \mathrm{U} / \mathrm{ml}) \times$ fasting glucose $(\mathrm{mmol} / \mathrm{l}) / 22 \cdot 5 ; \quad$ Quicki=1/(log (fasting insulin $(\mu \mathrm{U} / \mathrm{ml}))+\log$ (fasting glucose $(\mathrm{mg} / \mathrm{dl}))$ ).

\section{Zygosity}

MZ and DZ twin status was ascertained by nineteen questions covered in the maternal questionnaire. These questions were adapted from a validated Taiwan twin similarity questionnaire $^{(20)}$ and based on other related studies ${ }^{(21)}$, which asked about the children's degree of physical similarity and frequency of identity confusion. The accuracy rate of the validated questionnaire was over $95 \%$ for self-reports of adolescent twins and their mothers compared with results based on DNA diagnosis ${ }^{(20)}$. Only one twin pair was asked to answer these questions directly because their mother did not answer them.

\section{Statistical analysis}

Descriptive analyses were conducted to calculate mean values and standard deviations for all continuous phenotypes. Fasting serum glucose, insulin, Homa-IR and the Quicki index were continuous variables, and were summarised as means with standard errors. ANOVA was conducted to compare the differences between two or more groups for all continuous variables. Similarity was estimated by intra-class correlations (ICC) in $\mathrm{MZ}$ and DZ, which were calculated from a random-effects one-way ANOVA model. Shrout-Fleiss ICC were calculated for MZ and DZ twins separately using SAS 9.2 (SAS Institute).

Second, the data were analysed using quantitative genetic models for twin data. Twin studies are based on the assumption that MZ twins are genetically identical at the sequence level, and DZ twins have $50 \%$ of their genes shared by identical-by-descent. Thus, differences between MZ co-twins may be due to environmental effects, whereas differences between DZ co-twins are due to genetic and environmental effects. Under the above assumptions, four sources of variation can be interpreted as latent and standardised variance components using the structural equation models: additive genetic (A, additive effects of genes at multiple, independent genes), non-additive genetic (D, interactions between alleles at the same locus (dominance) or on different loci (epistasis)), common environmental (C, environmental effects shared by twins reared in the same family) and unique environmental effects ( $\mathrm{E}$, environmental effects unique to the individual). MZ pairs are assumed to share the same $A$ and $D$ genetic variances; $\mathrm{DZ}$ pairs are assumed to share one-half of the additive variance and one-quarter of the dominance variance. The $\mathrm{C}$ variance is assumed to be the same for both $\mathrm{MZ}$ and $\mathrm{DZ}$ twin pairs. Maximum likelihood methods were used to fit this general model and to estimate heritability of glucose, insulin, Homa-IR and the Quicki index. Heritability, which represents the proportion of variation in a quantitative trait due to genetic variation, was defined as the proportion of genetic variance to total phenotypic variance. Models were fit using Mx statistical package ${ }^{(22)}$. 
Third, we started the genetic modelling by running univariate models for glucose, insulin, Homa-IR and the Quicki index separately to estimate genetic and environmental influences and to find the best model for each trait. A likelihood ratio test (LRT) was used to test whether a significantly poorer fit was obtained when removing parameters. The LRT should follow a $\chi^{2}$ distribution, where a small $\chi^{2}$ and a high $P$ value indicate a good fit. Parsimony was assessed by means of the Akaike information criterion, and the model with the lowest Akaike information criterion was selected as the most parsimonious model. Estimates of variance components were derived from the best-fitting model and presented with 95\% CI.

Fourth, we then estimated pairwise genetic and environmental correlations between these quantitative phenotypes using full Cholesky decomposition. Genetic correlation is an estimate of the additive genetic effect that is shared between the pair of traits. For example, height and weight could both be heritable, but their genetic correlation can tell you whether they are likely to share the same genes. In this context, the correlations between dietary fat and each quantitative phenotype (glucose, insulin, Homa-IR and the Quicki index) were partitioned into an additive genetic correlation $\left(r_{\mathrm{A}}\right)$, which represents common genes controlling these phenotypes, and an environmental correlation $\left(r_{\mathrm{E}}\right)$, representing non-genetic, environmental factors common to dietary fat intake and the quantitative phenotypes. This bivariate heritability, which is a measure of the extent to which shared genetic influence generates a correlation between two traits, was calculated using the multivariate analysis module in the MX package.

Finally, a gene-diet interaction model was fit. The moderator factor (dietary fat intake) was denoted as $M$. This factor is presumed to affect the mean trait value $\left(\beta_{M}\right)$ but also modify the effects of genetic $\left(\beta_{X}\right)$ and environmental factors $\left(\beta_{Y}\right.$ and $\left.\beta_{Z}\right)$ on the trait. This model implies that dietary fat can affect the four quantitative phenotypes (glucose, insulin, Homa-IR and the Quicki index) and their variances

\section{Results}

Characteristics of dietary fat, insulin, glucose and insulin resistance

There were no systematic differences in means and variances of dietary fat intake, fasting serum glucose, fasting insulin, Homa-IR and the Quicki index between the MZ and the DZ twins (Table 1). The within-pair ICC for all traits were higher among MZ twins than among DZ twins, indicating that genetic factors are more important for glucose, insulin, Homa-IR and the Quicki index. The DZ correlation for dietary fat was nearly half of the corresponding $\mathrm{MZ}$ correlation, suggesting no presence of dominance (D) effects. Correlations for insulin, Homa-IR and the Quicki index within opposite-sex pairs were lower than within same-sex DZ pairs, suggesting the possible presence of sex-specific genetic effects (Table 1).

\section{Correlations between dietary fat and fasting serum glucose, insulin, homoeostasis model of assessment-insulin resistance and the Quicki index}

Dietary fat intake was significantly positively (but weakly) correlated with glucose $(r 0 \cdot 101, P=0 \cdot 013)$, insulin $(r 0 \cdot 157$, $P=0.001)$ and Homa-IR $(r-0.163, P<0.001)$, whereas dietary fat intake was negatively correlated with the Quicki index $(r-0.163, P<0.001)$ in the entire sample. To test for possible sex difference, analyses of the trait relationships were also conducted separately by sex. We observed similar patterns of correlation in female twins, but no significant correlations among male twins were found (Table 2).

\section{Genetic and environmental correlations between these traits}

We started our genetic modelling by estimating the best model for dietary fat intake, fasting serum glucose, insulin, Homa-IR and the Quicki index. The additive genetic/specific environment (AE) model offered the best fit for all of these traits: dropping common environmental (C) effect from the model had virtually no effect on model fit either. Fig. 1 summarises the proportions of phenotypic variance for all the traits explained by additive genetic and unique environmental factors under the best-fitting AE model. Estimated heritabilities for dietary fat intake, fasting serum glucose, insulin, Homa-IR and the Quicki index were 52, 70, 70, 63 and 55\%, respectively.

Fig. 1 also shows the model-based genetic and environmental correlations for these traits, as estimated from Cholesky models. The bivariate heritability is a measure of the extent to which shared genetic factors may influence two quantitative phenotypes and generates an estimated correlation for two traits. Our results showed that dietary fat intake and fasting serum insulin were significantly correlated $(r 0 \cdot 157)$, with a genetic correlation $\left(r_{\mathrm{A}} 0 \cdot 20 ; 95 \%\right.$ CI $\left.0.08,0.43\right)$. Dietary fat intake and glucose

Table 1. Characteristics of dietary fat intake, fasting blood glucose and insulin, and insulin resistance in Chinese child twins ( $n$ 622) (Mean values with their standard errors and intra-class correlations (ICC))

\begin{tabular}{|c|c|c|c|c|c|c|c|c|c|c|c|c|c|c|c|c|c|c|c|c|}
\hline & \multirow[b]{2}{*}{$n$} & \multicolumn{2}{|c|}{ Age (years) } & \multicolumn{2}{|c|}{ BMI $\left(\mathrm{kg} / \mathrm{m}^{2}\right)$} & \multicolumn{3}{|c|}{ Dietary fat (as $\%$ of energy) } & \multicolumn{3}{|c|}{ Glucose $(\mathrm{mmol} / \mathrm{l})$} & \multicolumn{3}{|c|}{ Insulin $(\mu \mathrm{U} / \mathrm{ml})$} & \multicolumn{3}{|c|}{ Homa-IR } & \multicolumn{3}{|c|}{ Quicki } \\
\hline & & Mean & SE & Mean & SE & Mean & SE & ICC & Mean & SE & ICC & Mean & SE & ICC & Mean & SE & ICC & Mean & SE & ICC \\
\hline All & 622 & $11 \cdot 31$ & 2.51 & $16 \cdot 63$ & 2.96 & 27.03 & 0.27 & 0.353 & 4.81 & 0.02 & 0.136 & $7 \cdot 81$ & $0 \cdot 17$ & 0.618 & 1.69 & 0.04 & 0.573 & 0.16 & 0.01 & 0.536 \\
\hline $\mathrm{MZ}$ & 324 & 11.55 & 2.51 & $16 \cdot 75$ & 3.01 & $26 \cdot 58$ & 0.36 & 0.482 & 4.78 & 0.02 & 0.144 & 7.64 & 0.23 & 0.762 & 1.64 & 0.05 & 0.686 & 0.16 & 0.01 & 0.615 \\
\hline DZ & 298 & 11.04 & 2.50 & 16.50 & 2.76 & 27.53 & 0.40 & 0.227 & 4.85 & 0.03 & 0.129 & 7.99 & 0.25 & 0.462 & 1.75 & 0.06 & 0.445 & 0.16 & 0.00 & 0.437 \\
\hline SDZ & 162 & 11.09 & 2.56 & $16 \cdot 31$ & 2.74 & $26 \cdot 8$ & 0.61 & 0.217 & 4.83 & 0.04 & 0.100 & 7.69 & 0.32 & 0.537 & 1.68 & 0.08 & 0.500 & 0.16 & 0.00 & 0.474 \\
\hline ODZ & 136 & 10.97 & 2.43 & 16.74 & 2.77 & 28.38 & 0.50 & 0.224 & 4.87 & 0.03 & 0.159 & 8.35 & 0.40 & 0.374 & 1.84 & 0.10 & 0.373 & 0.16 & 0.00 & 0.384 \\
\hline
\end{tabular}


Table 2. Pearson's partial correlation between dietary fat intake and fasting blood insulin and glucose levels among Chinese child twins ( $n$ 622), in all and by sex

(Pearson's partial correlation coefficients with their standard errors)

\begin{tabular}{|c|c|c|c|c|c|c|c|c|c|}
\hline & \multicolumn{3}{|c|}{ All $(n$ 622)* } & \multicolumn{3}{|c|}{ Males $(n$ 298)† } & \multicolumn{3}{|c|}{ Females $(n$ 322) $\dagger$} \\
\hline & Coefficient & $\mathrm{SE}$ & $P$ & Coefficient & $\mathrm{SE}$ & $P$ & Coefficient & SE & $P$ \\
\hline Glucose (mmol/l) & $0 \cdot 10$ & 0.08 & 0.013 & 0.01 & 0.01 & 0.952 & 0.15 & 0.08 & 0.005 \\
\hline Insulin $(\mu \mathrm{U} / \mathrm{ml})$ & $0 \cdot 16$ & 0.10 & 0.001 & 0.05 & 0.05 & 0.396 & 0.26 & 0.11 & $<0.001$ \\
\hline Homa-IR & $0 \cdot 16$ & 0.11 & $<0.001$ & 0.05 & 0.04 & 0.416 & 0.28 & 0.12 & $<0.001$ \\
\hline Quicki & -0.16 & 0.12 & $<0.001$ & -0.05 & 0.03 & 0.407 & -0.27 & 0.12 & $<0.001$ \\
\hline
\end{tabular}

Homa-IR, homoeostasis model assessment-insulin resistance; Quicki, quantitative insulin sensitivity check index.

* Adjusted for age, sex, BMl, total energy.

$\dagger$ Adjusted for age, BMI, total energy.

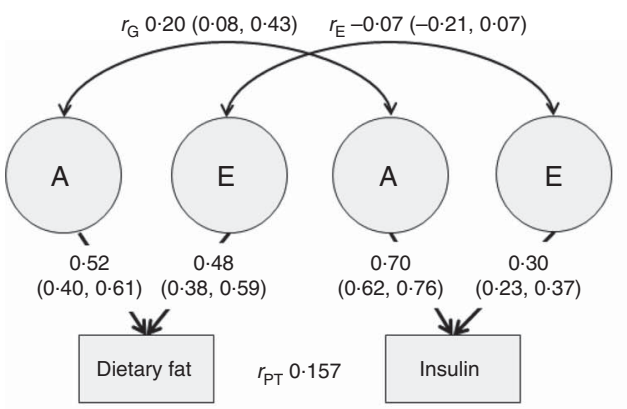

Bivariate heritability $=0 \cdot 121$

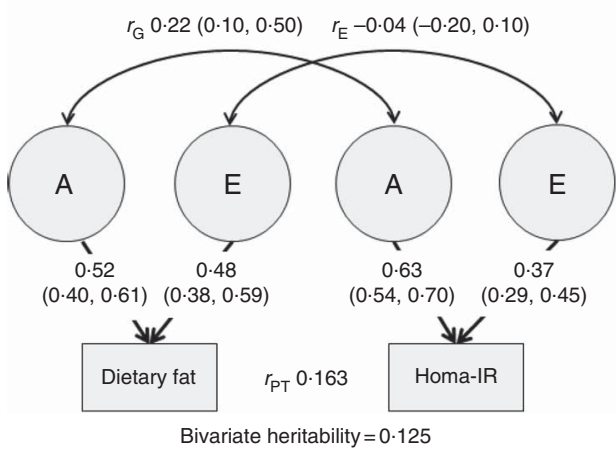

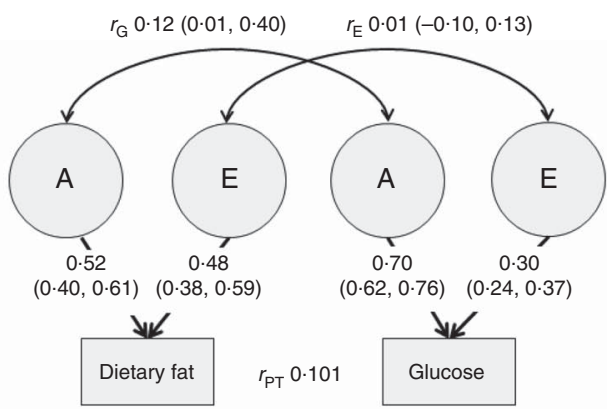

Bivariate heritability $=0.073$



Bivariate heritability $=0 \cdot 118$

Fig. 1. Bivariate association analysis of dietary fat and glucose, insulin, homoeostasis model of assessment-insulin resistance (Homa-IR) and the quantitative insulin sensitivity check index (Quicki) among Chinese child twins ( $n$ 622). $r_{\mathrm{G}}$, Additive genetic (A) correlation; $r_{\mathrm{E}}$, unique environmental $(\mathrm{E})$ correlation; $r_{\mathrm{PT}}$, phenotypic correlation.

$\left(r_{\mathrm{A}} 0 \cdot 12 ; 95 \%\right.$ CI $\left.0 \cdot 01,0 \cdot 40\right)$, Homa-IR $\left(r_{\mathrm{A}} 0 \cdot 22 ; 95 \%\right.$ CI $0 \cdot 10$, $0 \cdot 50)$ and the Quicki index $\left(r_{\mathrm{A}}-0 \cdot 22 ; 95 \% \mathrm{CI}-0 \cdot 40,0 \cdot 04\right)$ all showed strong estimated genetic correlations. The environmental correlation was not significant $\left(\mathrm{r}_{E}-0 \cdot 07 ; 95 \%\right.$ CI $-0 \cdot 21$, 0.07). The calculated bivariate heritabilities of dietary fat and insulin, fasting glucose, Homa-IR and the Quicki index were $0 \cdot 121,0 \cdot 073,0 \cdot 125$ and $0 \cdot 118$, respectively (Fig. 1).

\section{Gene-environmental interactions on these type 2 diabetes-related traits}

Finally, we tested dietary fat intake as a moderator of genetic and/or environmental effects on quantitative measures of T2Drelated traits including glucose, insulin, Homa-IR and the Quicki index (Table 3). Fat intake significantly modified additive genetic effects on these T2D-related traits. The estimated additive genetic variance decreased, whereas the unique environmental variance increased with increasing dietary fat in determining insulin and the Quicki index (Fig. 2). This indicates that genetic factors play a less important role in determining insulin levels and the Quicki index in subjects with high dietary fat intakes compared with lower intakes. In contrast, the estimated additive genetic variance was increased, whereas the unique environmental variance decreased with increasing dietary fat intake from analyses of glucose and Homa-IR (Fig. 2). This suggests that genetic factors may play a more important role in determining glucose and Homa-IR levels among subjects with high dietary fat intakes compared with lower intakes. 


\section{Discussion}

The present study provided unique insights about how dietary fat intake is associated with quantitative phenotypes related to T2D (including insulin, glucose, Homa-IR and the Quicki index) in Chinese children. To our knowledge, this is the first report to estimate genetic and environmental correlations between dietary fat intake and T2D-related traits in children. Our results suggest that genetic factors contribute to a significant proportion of the total variance in IR, IS and fat intake among Chinese

Table 3. Moderating effect of dietary fat intake on genetic and specific environmental variances of fasting insulin and glucose and insulin resistance among Chinese child twins $(n 622)^{*}$

(Additive genetic modifications (A), specific environmental modifications (E) and $95 \%$ confidence intervals)

\begin{tabular}{lrcrr}
\hline & \multicolumn{4}{c}{ Moderating effects } \\
\cline { 2 - 5 } $\begin{array}{l}\text { Dietary fat intake } \\
\text { (as \% of total energy) }\end{array}$ & $\mathrm{A}$ & $95 \% \mathrm{Cl}$ & $\mathrm{E}$ & $95 \% \mathrm{Cl}$ \\
\hline Glucose $(\mathrm{mmol} / \mathrm{l})$ & -0.33 & $-0.45,-0.20$ & 0.19 & $0.07,0.37$ \\
Insulin $(\mu \mathrm{U} / \mathrm{ml})$ & 0.19 & $0.04,0.36$ & 0.07 & $-0.05,0.17$ \\
Homa-IR & 0.16 & $0.02,0.42$ & -0.22 & $-0.46,-0.14$ \\
Quicki & -0.22 & $-0.46,-0.07$ & 0.07 & $-0.02,0.22$ \\
\hline
\end{tabular}

Homa-IR, homoeostasis model assessment-insulin resistance; Quicki, quantitative insulin sensitivity check index.

* Additive genetic and specific correlations were based on re-parameterisation of Cholesky decomposition model. children. Phenotypic correlations between dietary fat intake and quantitative traits related to $\mathrm{T} 2 \mathrm{D}$ are mediated by common genetic factors.

Our results provide further evidence for the role of genes in controlling IR, IS and glucose metabolism. Our results are consistent with previous evidences showing estimated heritability for peripheral IS $\left(b^{2} 0.53\right)$ and non-oxidative glucose metabolism ( $\left.b^{2} 0.50\right)$ in young people, supporting the notion that there are both genetic and environmental aetiological factors controlling insulin action and non-oxidative glucose metabolism $^{(23)}$. Our findings lend further support for a major genetic component in the aetiology of insulin secretion.

Previous epidemiological studies have found that dietary fat intake is positively associated with T2D risk, blood insulin and glucose $\mathrm{e}^{(24,25)}$. We previously found that high total fatty acids were positively associated with diabetes-related quantitative traits in Chinese ${ }^{(26,27)}$. Our findings in Chinese child twins further supported this result. Dietary fat intake was significantly positively correlated with glucose, insulin and Homa-IR levels. The potential mechanisms through which dietary fat influence IS levels were elucidated $^{(28-33)}$. In rodents, a high-fat diet increased the concentration of diacylglycerol in muscles and activated novel protein kinase $\mathrm{C}$ (PKC) ${ }^{(32)}$. Thus, the accumulation of diacylglycerol impaired activation of the insulin receptor ${ }^{(30)}$. Similarly, infusion of lipid for $5 \mathrm{~h}$ caused IR in muscles associated with accumulation of intracellular
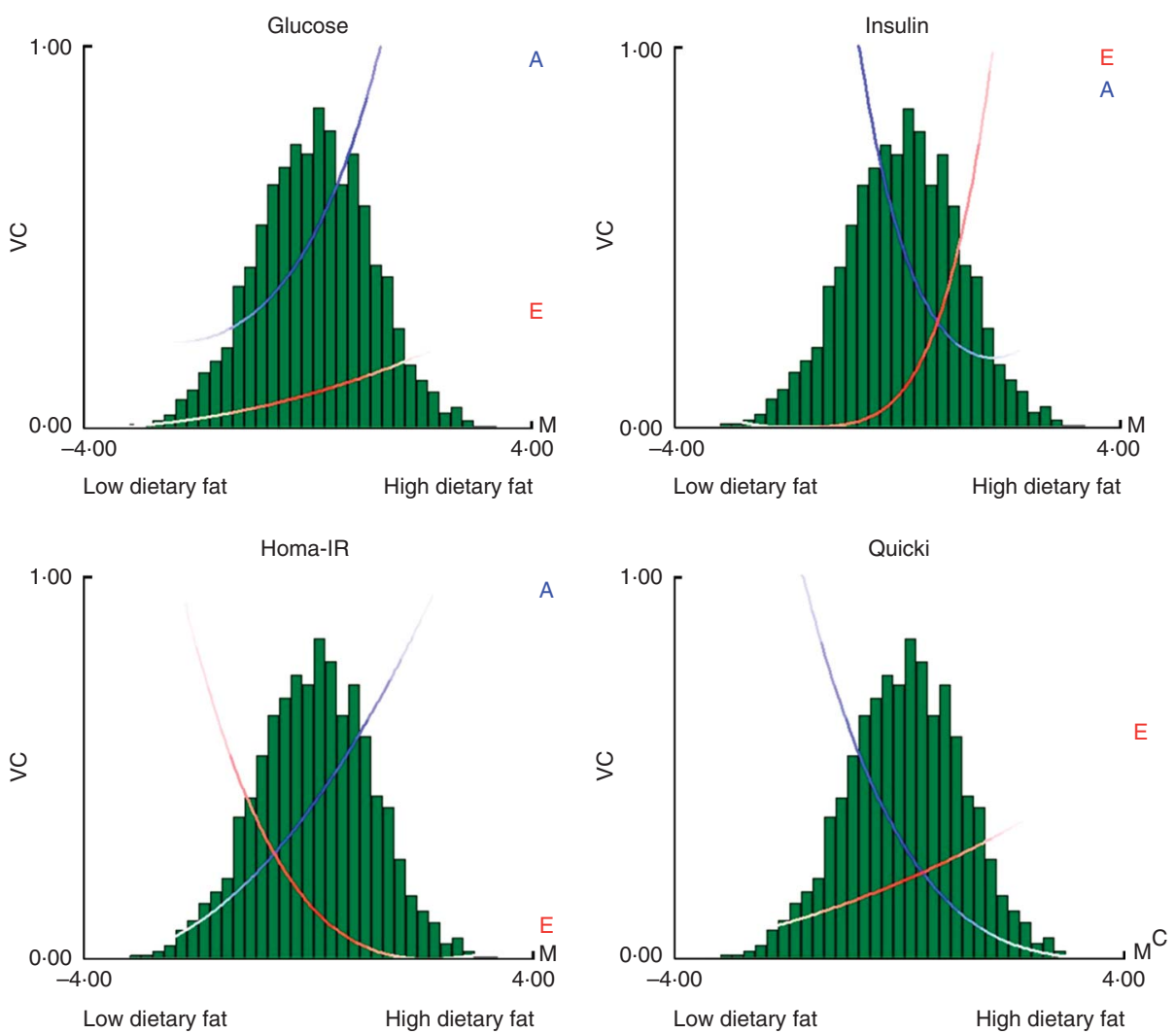

Fig. 2. Change in additive genetic $(A,-)$ and unique environmental variance $(E,-\longrightarrow)$ of glucose and insulin with increasing levels of dietary fat among Chinese child twins ( $n$ 622). Dietary fat significantly modified additive genetic effects on glucose, insulin, homoeostasis model of assessment-insulin resistance (Homa-IR) and the quantitative insulin sensitivity check index (Quicki) levels. High dietary fat intake may accentuate the genetic influence in determining glucose and Homa-IR. In contrast, high dietary fat intake may attenuate the genetic influence in determining insulin and Quicki. 
diacylglycerol and specific activation of $\mathrm{PKC}^{(34)}$. A study in mice without $\operatorname{PKC} \theta$ showed the importance of activation of novel PKC and serine phosphorylation of insulin receptor substrate 1 (IRS1) for development of $\mathrm{IR}^{(33)}$. On the other hand, IR can also be attributed to lipid-induced defects in the insulin signalling pathway originating from a reduction in tyrosine phosphorylation of IRS1, which plays a key role in transmitting signals from the insulin and insulin-like growth factor-1 receptors to intracellular pathways phosphoinositide 3 kinase and protein kinase $\mathrm{B}$ (PI3K/Akt) and Erk mitogen-activated protein (MAP) kinase pathways ${ }^{(35)}$.

Homa-IR and fasting insulin levels showed high genetic correlations. Homa-IR and IS yielded moderate estimated genetic correlations, suggesting that these two quantitative traits are influenced, at least in part, by different genes ${ }^{(36)}$. In addition, the correlations between dietary fat intake and T2D-related traits may also be mediated by shared genetic influences. Thus, to elucidate the mechanism, we conducted a bivariate analysis using Cholesky models in the present study. The estimated bivariate heritability reflects the extent to which shared genetic factors underlie a correlation between two quantitative traits. Our results showed that dietary fat intake and fasting serum insulin were significantly correlated, with common genetic factors creating a correlation. Overall $77 \%$ of this phenotypic correlation was mediated by the shared genetic factors. In the same way, genetic factors also appear to underlie the correlations between dietary fat intake and glucose, Homa-IR and Quicki levels. Our results provide further evidence of some role of genetic factors in mediating phonotypic correlations between dietary fat and T2D-related traits.

Considering the evidence for gene-environment interactions in the aetiology of diabetes, we further investigated the effects of genetic and environmental interactions on T2D-related traits. The present study indicates that dietary fat significantly modified additive genetic effects on glucose, insulin, Homa-IR and Quicki levels. High dietary fat intake may accentuate the genetic influence in determining blood glucose and Homa-IR. In contrast, high dietary fat intake may attenuate the genetic influence in determining blood insulin and the Quicki index. Our findings can be supported by previous evidences that suggest a possible interaction between Pro12Ala polymorphism of PPARG2 (a recognised candidate gene for T2D) and dietary MUFA, such that obese people with the Ala-12 allele have higher Homa-IR values, especially if their intake of MUFA is low ${ }^{(37)}$. Dietary fat intake modifies the effects of $-514(\mathrm{C} / \mathrm{T})$ polymorphism on HDL-cholesterol concentrations and its subclasses. Specifically, in the Framingham Study, TT subjects displayed impaired adaptation to high animal fat diets, which could in turn result in higher cardiovascular and diabetes risk $^{(38)}$. Therefore, our study together with previous studies further supported the hypothesis that IR results from the interaction between genetic and environmental factors.

In conclusion, genetic factors contribute to a significant proportion of the total variance in T2D-related traits among Chinese children. These associations between dietary fat intake and T2D-related traits are mostly mediated by genetic influences. Future longitudinal studies are needed to test the related causal relationships.

\section{Acknowledgements}

The authors thank all the study participants for their support. The authors thank Drs Zhu Li, Hua Chen, Yexuan Tao and Xia Liu for their efforts to develop the study as well as Dr Hong Xue and Vivian Wang for their assistance in managing some related research human subject protection issues. The authors also thank Drs Duo Li and Mark L. Wahlqvist from Zhejiang University for their comments on an early draft of the manuscript to help improve it. The content of this article is solely the responsibility of the authors and does not necessarily represent the official views of the funder.

The project including data collection and analysis was supported in part by research grants from the National Institutes of Institute of Diabetes and Digestive and Kidney (P60DK0079637), Health Eunice Kennedy Shriver National Institute of Child Health and Human Development and the Office of the Director, National Institutes of Health (U54 HD070725), the Nestle Foundation, and the Faculty Innovation Fund and Procter \& Gamble Fellowship from the Johns Hopkins Bloomberg School of Public Health. The funders had no role in the design, analysis or writing of this article.

T. H. and Y. W. formulated the research question(s); T. H., T. B. and Y. W. designed the study; T. H., H. L. and W. Z. carried out the study; T. H. analysed the data and wrote the article.

The authors declare that there are no conflicts of interest.

\section{References}

1. Wareham NJ, Franks PW \& Harding AH (2002) Establishing the role of gene-environment interactions in the etiology of type 2 diabetes. Endocrinol Metab Clin North Am 31, 553-566.

2. Hu FB, van Dam RM \& Liu S (2001) Diet and risk of type II diabetes: the role of types of fat and carbohydrate. Diabetologia 44, 805-817.

3. Lopez S, Bermudez B, Pacheco YM, et al. (2008) Distinctive postprandial modulation of beta cell function and insulin sensitivity by dietary fats: monounsaturated compared with saturated fatty acids. Am J Clin Nutr 88, 638-644.

4. van Dam RM, Stampfer M, Willett WC, et al. (2002) Dietary fat and meat intake in relation to risk of type 2 diabetes in men. Diabetes Care 25, 417-424.

5. Pedrini MT, Niederwanger A, Kranebitter M, et al. (2006) Postprandial lipaemia induces an acute decrease of insulin sensitivity in healthy men independently of plasma NEFA levels. Diabetologia 49, 1612-1618.

6. Huang T, Wahlqvist ML \& Li D (2010) Docosahexaenoic acid decreases plasma homocysteine via regulating enzyme activity and mRNA expression involved in methionine metabolism. Nutrition 26, 112-119.

7. Vessby B, Tengblad S \& Lithell H (1994) Insulin sensitivity is related to the fatty-acid composition of serum-lipids and skeletal-muscle phospholipids in 70-year-old men. Diabetologia 37, 1044-1050.

8. Storlien LH, Baur LA, Kriketos AD, et al. (1996) Dietary fats and insulin action. Diabetologia 39, 621-631.

9. Maedler K, Oberholzer J, Bucher P, et al. (2003) Monounsaturated fatty acids prevent the deleterious effects of palmitate and high glucose on human pancreatic beta-cell turnover and function. Diabetes 52, 726-733.

10. Saxena R, Voight BF, Lyssenko V, et al. (2007) Genome-wide association analysis identifies loci for type 2 diabetes and triglyceride levels. Science 316, 1331-1336. 
11. Saxena R, Voight BF, Zeggini E, et al. (2008) Meta-analysis of genome-wide association data and large-scale replication identifies several additional susceptibility loci for type 2 diabetes. Diabetes 57, A41-A41.

12. Manolio TA, Collins FS, Cox NJ, et al. (2009) Finding the missing heritability of complex diseases. Nature 461, 747-753.

13. Ruchat SM, Elks CE, Loos RJ, et al. (2009) Evidence of interaction between type 2 diabetes susceptibility genes and dietary fat intake for adiposity and glucose homeostasisrelated phenotypes. J Nutrigenet Nutrigenomics 2, 225-234.

14. Huang T, Huang J, Qi Q, et al. (2014) PCSK 7 genotype modifies effect of a weight-loss diet on 2-year changes of insulin resistance: the POUNDS LOST trial. Diabetes Care 38, 439-444.

15. Qi Q, Xu M, Wu H, et al. (2013) IRS1 genotype modulates metabolic syndrome reversion in response to 2-year weightloss diet intervention: the POUNDS LOST trial. Diabetes Care 36, 3442-3447.

16. Mattei J, Qi Q, Hu FB, et al. (2012) TCF7L2 genetic variants modulate the effect of dietary fat intake on changes in body composition during a weight-loss intervention. Am J Clin Nutr 96, 1129-1136.

17. Qi Q, Bray GA, Smith SR, et al. (2011) Insulin receptor substrate 1 gene variation modifies insulin resistance response to weight-loss diets in a 2-year randomized trial: the Preventing Overweight Using Novel Dietary Strategies (POUNDS LOST) trial. Circulation 124, 563-571.

18. Wang W (2009) Reproducibility and Validation of a Food Frequency Questionnaire Among Children and Adolescents in Beijing. Beijing: Capital Institute of Pediatrics.

19. Yang YX (2004) China Food Composition. Beijing: Peking University Medical Publisher.

20. Chen WJ, Chang HW, Wu MZ, et al. (1999) Diagnosis of zygosity by questionnaire and polymarker polymerase chain reaction in young twins. Behav Genet 29, 115-123.

21. Ooki S \& Asaka A (2004) Zygosity diagnosis in young twins by questionnaire for twins' mothers and twins' self-reports. Twin Res 7, 5-12.

22. Neale MC (2003) Mx: Statistical Modeling. Richmond, VA: Department of Psychiatry.

23. Poulsen P, Levin K, Petersen I, et al. (2005) Heritability of insulin secretion, peripheral and hepatic insulin action, and intracellular glucose partitioning in young and old Danish twins. Diabetes 54, 275-283.

24. Vessby B (2000) Dietary fat and insulin action in humans. Br J Nutr 83, Suppl. 1, S91-S96.

25. Mayer-Davis EJ, Monaco JH, Hoen HM, et al. (1997) Dietary fat and insulin sensitivity in a triethnic population: the role of obesity. The Insulin Resistance Atherosclerosis Study (IRAS). Am J Clin Nutr 65, 79-87.
26. Huang T, Bhulaidok S, Cai ZZ, et al. (2010) Plasma phospholipids $n-3$ polyunsaturated fatty acid is associated with metabolic syndrome. Mol Nutr Food Res 54, 1628-1635.

27. Huang T, Wahlqvist ML, Xu TC, et al. (2010) Increased plasma $n-3$ polyunsaturated fatty acid is associated with improved insulin sensitivity in type 2 diabetes in China. Mol Nutr Food Res 54, S112-S119.

28. Galbo T, Perry RJ, Jurczak MJ, et al. (2013) Saturated and unsaturated fat induce hepatic insulin resistance independently of TLR-4 signaling and ceramide synthesis in vivo. Proc Natl Acad Sci U S A 110, 12780-12785.

29. Erion DM \& Shulman GI (2010) Diacylglycerol-mediated insulin resistance. Nature Med 16, 400-402.

30. Samuel VT, Petersen KF \& Shulman GI (2010) Lipid-induced insulin resistance: unravelling the mechanism. Lancet $\mathbf{3 7 5}$, 2267-2277.

31. Hoehn KL, Salmon AB, Hohnen-Behrens C, et al. (2009) Insulin resistance is a cellular antioxidant defense mechanism. Proc Natil Acad Sci U S A 106, 17787-17792.

32. SchmitzPeiffer C, Browne CL, Oakes ND, et al. (1997) Alterations in the expression and cellular localization of protein kinase $\mathrm{C}$ isozymes epsilon and theta are associated with insulin resistance in skeletal muscle of the high-fat-fed rat. Diabetes 46, 169-178.

33. Kim JK, Fillmore JJ, Sunshine MJ, et al. (2004) PKC-theta knockout mice are protected from fat-induced insulin resistance. J Clin Invest 114, 823-827.

34. Griffin ME, Marcucci MJ, Cline GW, et al. (1999) Free fatty acid-induced insulin resistance is associated with activation of protein kinase $\mathrm{C}$ theta and alterations in the insulin signaling cascade. Diabetes 48, 1270-1274.

35. Yu CL, Chen Y, Cline GW, et al. (2002) Mechanism by which fatty acids inhibit insulin activation of insulin receptor substrate-1 (IRS-1)-associated phosphatidylinositol 3-kinase activity in muscle. J Biol Chem 277, 50230-50236.

36. Rasmussen-Torvik LJ, Pankow JS, Jacobs DR, et al. (2007) Heritability and genetic correlations of insulin sensitivity measured by the euglycaemic clamp. Diabet Med 24, 1286-1289.

37. Soriguer F, Morcillo S, Cardona F, et al. (2006) Pro12Ala polymorphism of the PPARG2 gene is associated with type 2 diabetes mellitus and peripheral insulin sensitivity in a population with a high intake of oleic acid. J Nutr $\mathbf{1 3 6}$, $2325-2330$.

38. Ordovas JM, Corella D, Demissie S, et al. (2002) Dietary fat intake determines the effect of a common polymorphism in the hepatic lipase gene promoter on high-density lipoprotein metabolism - evidence of a strong dose effect in this genenutrient interaction in the Framingham Study. Circulation 106, 2315-2321. 